\title{
Design and development of a hybrid artificial bee colony algorithm for the environmental vehicle routing problem
}

\begin{abstract}
The vehicle routing problem (VRP) is a critical and vital problem in logistics for the design of an effective and efficient transportation network, within which the capacitated vehicle routing problem (CVRP) has been widely studied for several decades due to the practical relevance of logistics operation. However, CVRP with the objectives of minimizing the overall travelling distance or the travelling time cannot meet the latest requirements of green logistics, which concern more about the influence on the environment. This paper studies CVRP from an environmental perspective and introduces a new model called environmental vehicle routing problem (EVRP) with the aim of reducing the adverse effect on the environment caused by the routing of vehicles. In this research, the environmental influence is measured through the amount of the emission carbon dioxide, which is a widely acknowledged criteria and accounts for the major influence on environment. A hybrid artificial bee colony algorithm (ABC) is designed to solve the EVRP model, and the performance of the hybrid algorithm is evaluated through comparing with well-known CVRP instances. The computational results from numerical experiments suggest that the hybrid ABC algorithm outperforms the original $\mathrm{ABC}$ algorithm by $5 \%$ on average. The transformation from CVRP to EVRP can be recognized through the differentiation of their corresponding optimal solutions, which provides practical insights for operation management in green logistics.
\end{abstract}

Keywords: Green logistics, Capacitated vehicle routing problem, Artificial bee colony algorithm, Environmental influence, Environmental vehicle routing problem, $\mathrm{CO}_{2}$ emission

\section{Introduction}

Due to increasingly serious environmental deterioration, sustainable development is becoming a vital issue facing various enterprises (Akyelken, 2011). Both environmental regulations and customer pressures have driven enterprises to adopt more environment-friendly operations (Dobers et al., 2013). In the logistics area, green logistics has attracted more and more attention as an exemplar of sustainable development (Sheu et al., 2005). Environmental issues can affect various logistics decisions, such as warehouse location, material sourcing, modal selection, 
transportation management and so on (Suzuki, 2011; Tancrez et al., 2012; Wu and Dunn, 1995). Transportation is the largest source of pollution in logistics. For example, in Canada, transportation accounted for $27 \%$ of greenhouse gas (GHG) emission in 2007 (Environment Canada, 2009), and in the United States, the transportation sector contributed $28 \%$ of national GHG emission (US EPA, 2009). Amongst various transportation modes, medium to heavy-duty diesel vehicles account for around one-third of GHG emissions from transportation (Elhedhli and Merrick, 2012). Therefore, more effort should be devoted to handle transportation management in logistics with the objective of minimizing the environmental impact (Salimifard et al., 2012).

The vehicle routing problem (VRP) has been extensively studied ever since its introduction. The initial idea of the VRP is to deliver a certain amount of goods to a set of customers with known demands through a number of vehicles to achieve the objective of minimizing cost. VRP plays a pivotal role in the design of distribution networks and has also been applied in the agricultural field (Bochtis and Sørensen, 2010). VRP has been extended to different specific problems, such as Capacitated VRP (CVRP) concerning the limited capacity of vehicles, VRP with time windows (VRPTW) considering the delivering goods within a time window, multi-depot VRP (m-VRP), VRP with simultaneous pick-up and delivery (VRPSPD) and so on. Each of these has different objectives, constraints and application backgrounds. Surveys and reviews of classical and derived VRP model can be found by Toth and Vigo (2002), Golden et al. (2008), and Hoff et al. (2010). Among the various types of VRPs, CVRP is the most well-known and practical one and has been extensively studied by academic researchers. Traditional VRP is designed with the economic objective of minimizing the cost while designing the route and scheduling vehicles. The cost is normally represented in the form of travelling distance or time. However, in recent years, the objective in solving VRP does not exclusively consider the economic needs.

In green logistics, the VRP involving environmental issue has also been studied from different perspectives by many researchers (Lin et al., 2013). For example, Kara et al. (2007) firstly proposed an Energy Minimizing Vehicle Routing Problem (EMVRP), considering a new cost function based on distance and the vehicle load. However, the cost function was derived more from physical and mechanical analyses, which differ from practical situations. Xiao et al. (2012) extended the idea of EMVRP and proposed a fuel consumption optimization model for CVRP. In their research, they formulated a linear expression between fuel consumption rate and the weight of vehicles based on the analysis of past statistical data. Nevertheless, in their research, the load of each vehicle was not explicitly represented with the combination of the route traversed. In addition, in their numerical experiments, they assumed the full-load and empty-load fuel consumption rates as 2 and 1 for simplicity, which is not practical for real situations. In numerical experiments, we find that the settings of these two parameters can largely affect the construction of the final solution. Thus, in 
this research, we set the parameters in accordance with a practical case study by Ubeda et al. (2011), the $\mathrm{CO}_{2}$ emission rate per liter of fuel is $2.61 \mathrm{~kg} / \mathrm{l}$ in case of the diesel oil and the empty-load and full-load fuel consumption rates are as 0.296 and 0.390 respectively.

In this research, the emission of $\mathrm{CO}_{2}$ is employed as a measurement to formulate an environmental vehicle routing problem (EVRP) model. In contrast to the CVRP, the objective of the EVRP is to find the optimal solution with minimum environmental impact in terms of minimizing $\mathrm{CO}_{2}$ emissions. For the purpose of generalization, the emission of $\mathrm{CO}_{2}$ is determined by the fuel consumption directly, either diesel or gasoline. The fuel consumption of one vehicle is subjected to three major factors, the travelling distance, the truckload and the travelling speed (Elhedhli and Merrick, 2012). Modeling the fuel consumption function depends on the transportation strategy. In the fundamental CVRP, we adopt a linear function relating fuel consumption and the load of vehicles (Xiao et al., 2012), wherein the speed is assumed to be constant. Apart from the three factors mentioned earlier, other factors like road conditions, traffic jams and weather may also affect the fuel consumption. Nevertheless, these factors are relatively insignificant, and occur in special cases; hence they are not included herein.

VRPs can be modeled as combinatorial optimization (CO) problems with multiple objectives, constraints and decision variables. Exact methods, such as Linear Programming (LP) and Branch-and-Bound (B\&B), are becoming less popular for solving $\mathrm{CO}$ problems, as they are either unable to solve complicated $\mathrm{CO}$ problems with large numbers of variables or it takes long time to find the solution for $\mathrm{CO}$ problems (Laporte, 1992). By contrast, meta-heuristic approaches are becoming increasingly popular as these approaches are approximate approaches, which suggest that they could find satisfactory solutions within an acceptable time instead of finding the optimal solution. Swarm intelligence, which was originally inspired by the collective behavior of natural insect colonies and animal societies, is a new branch of meta-heuristics, comparing with the evolutionary computations (Bonabeau et al., 1999). Swarm intelligent algorithms use approximate and non-deterministic strategies to effectively and efficiently explore and exploit the search space in order to find near-optimal solutions (Blum and Li, 2008; Blum and Merkle, 2008). Two essential properties, self-organization and division of labor, are necessary and sufficient to obtain swarm intelligent behavior. Self-organization relies on four basic properties; positive feedback, negative feedback, fluctuations and multiple interactions (Bonabeau et al., 1999), while division of labor indicates that different tasks are performed simultaneously by specialized individuals, and is believed to be more efficient than the sequential task performance by unspecialized individuals (Jeanne, 1986). The typical example of swarm intelligence is the behavior of bee colonies, which derives the introduction of the artificial bee colony (ABC) algorithm by Karaboga (2005). Both self-organization and labor division are clearly exemplified in 
$\mathrm{ABC}$ algorithm. The framework of $\mathrm{ABC}$ algorithm balances the effect of diversification and intensification effectively when searching the whole search space, which means the exploration of the whole search space and the exploitation of the promising area in the search space are well organized. Since the introduction of ABC algorithm, it has gained much popularity because of its robust mechanism and easy implementation (Akay and Karaboga, 2012; Karaboga et al., 2012). However, until now there are few studies of applying $\mathrm{ABC}$ algorithm into green logistics. Therefore, this research is a pioneering attempt for the integration of swarm intelligence and green logistics. Differing from the original $\mathrm{ABC}$ algorithm, we introduce a hybrid $\mathrm{ABC}$ algorithm by incorporating the evolutionary concept of genetic algorithm (GA) and the local search algorithm for EVRP. The computational performance of the proposed hybrid $\mathrm{ABC}$ algorithm is measured in numerical experiments, comparing with the original $\mathrm{ABC}$ algorithm and $\mathrm{GA}$, which shows that the hybrid $\mathrm{ABC}$ algorithm outperforms the other two algorithms, and can generate good solutions within acceptable computational time.

The contribution of this paper is two-fold. First, we introduced a new vehicle routing model (EVRP) taking account of the environmental influence. The environmental influence in EVRP is identified and quantified in terms of the emission of $\mathrm{CO}_{2}$, which is well acknowledged. And the $\mathrm{CO}_{2}$ emission is further computed by the fuel consumption, which is determined by various possible factors in transportation. In contrast with the CVRP, the proposed EVRP model is rather straight-forward and well perceived, without excessive assumptions or constraints and can directly inspire practitioners in realizing the importance of green transportation management. Second, the proposed hybrid ABC algorithm, which is proven to be effective and efficient in solving EVRP, is a pilot attempt of applying swarm intelligence into green logistics, which could facilitate the integrated study of green logistics and swarm intelligence. Apart from the above two aspects, the comparative studies in numerical experiments indicates the transformation of the optimal solutions from the situation of shortest travelling distance to the situation of minimum environmental influence, and provides practical managerial implications for decision-making in green logistics.

The rest of this paper is organized as follows. After a brief introduction about vehicle routing and environmental influence in section 1, we propose the model formulation of EVRP and elaborate on the explicit objectives and constraints in section 2. A hybrid $A B C$ algorithm with detailed explanation is proposed in Section 3 to solve the new EVRP. In section 4, we describe numerical experiments with well-acknowledged benchmark instances comparing the performance of the hybrid $\mathrm{ABC}$ algorithm and the GA algorithm, and analyze the differentiation between the optimal solutions for different situation. Finally, the conclusions and further work are drawn in section 5 . 


\section{Environmental vehicle routing model}

\subsection{Vehicle routing model}

VRP is normally modeled on a graph, which comprises a set of nodes and the associative edges. The set of nodes represent the $\operatorname{depot}(\mathrm{s})$ and customers, while the edges represent the routes among them. A number of vehicles depart from the $\operatorname{depot}(\mathrm{s})$, visit customers and return to the $\operatorname{depot}(\mathrm{s})$. The number of vehicles needed, the allocation of customers for vehicles and the optimal routes for vehicles are three intrinsic aspects of VRP. The settings and notations of VRP are described in table 1.

Table 1. The settings and notations of VRP

\begin{tabular}{|l|c|l|}
\hline \multirow{4}{*}{$\begin{array}{l}\text { Graph } \\
\text { parameters }\end{array}$} & Notations & Meanings \\
\cline { 2 - 3 } & $G=(V, A)$ & The graph \\
\cline { 2 - 3 } & $A=\{(i, j) \mid i, j \in V, i \neq j\}$ & The set of nodes in the graph \\
\cline { 2 - 3 } & $d_{i j}=d_{j i}(i, j \in V, i \neq j)$ & The distance between $i$ and $j$ \\
\cline { 2 - 3 } & $c_{i j}=\alpha * d_{i j}$ & The travelling cost on edge $(i, j)$ \\
\hline $\begin{array}{l}\text { Customer-related } \\
\text { parameter }\end{array}$ & $r_{i}(i=1,2, \cdots, n)$ & The customer demand \\
\hline $\begin{array}{l}\text { Vehicle-related } \\
\text { parameter }\end{array}$ & $Q$ & The vehicle capacity \\
\cline { 2 - 3 } & $m$ & \multicolumn{2}{|c|}{ The maximum number of vehicles } \\
\hline
\end{tabular}

In this research, the scenario of single depot and symmetric network is adopted, which means all the vehicles depart from and return to the same depot and the route between two nodes contains no directional information. In detail, node $i=0$ is the depot, and the other nodes $i=1,2, \cdots, n$ represent customers. The distance between node $i$ and node $j$ is represented as $d_{i j}(i, j \in V, i \neq j)$, and the symmetric network indicates that $d_{i j}=d_{j i}(i, j \in V, i \neq j)$. Customer demand is represented as $r_{i}(i=$ $1,2, \cdots, n)$, which is predetermined in this research. $m$ vehicles with same load capacity $Q$ are available. The travelling cost between customer $i$ and $j$ is illustrated as $c_{i j}$, and treated from a distance perspective as $c_{i j}=\alpha * d_{i j}$, in which $\alpha$ is the correlative coefficient depicting the proportion between economic cost and travelling distance. The above assumptions and notations are sufficient to construct a vehicle routing model with the objective of finding the shortest travelling distance. In practical situations, there might be some other constraints for the entities (i.e. depot, customers and vehicles), such as multiple depots, uncertain demand and time window for customers, and maximum allowed travelling distance for vehicles. 


\subsection{Environmental influence}

As we mentioned above, the environmental influence is externalized in terms of the $\mathrm{CO}_{2}$ emission. Therefore, the critical issue is to measure the amount of $\mathrm{CO}_{2}$ emission. In this research, we use $e_{i j}$ to denote the amount of $\mathrm{CO}_{2}$ emission when a vehicle travels from customer $i$ to customer $j$, and try to find the optimal routes with the least $\mathrm{CO}_{2}$ emission considering the balance between $c_{i j}$ and $e_{i j}$. The added settings and notations for environmental measurement are provided in table 2.

Table 2. The settings and notations for environmental measurement

\begin{tabular}{|l|c|l|}
\hline Parameter & Notations & Meanings \\
\hline \multirow{4}{*}{$\mathrm{CO}_{2}$ emission } & \multicolumn{1}{|c|}{$C E R$} & The $\mathrm{CO}_{2}$ emission rate \\
\cline { 2 - 3 } & $F C R$ & The fuel consumption rate \\
\cline { 2 - 3 } & $e_{i j}=C E R * F C R * d_{i j}$ & The amount of $\mathrm{CO}_{2}$ emission \\
\cline { 2 - 3 } Fuel consumption & $\rho_{0}$ & The empty-load $F C R$ \\
\cline { 2 - 3 } & $\rho^{*}$ & The full-load $F C R$ \\
\hline \multirow{3}{*}{ Vehicle load } & $\rho$ & The $F C R$ provided that load is $q$ \\
\cline { 2 - 3 } & $q$ & The real-time load of vehicle \\
\cline { 2 - 3 } & $q_{i j k}$ & The initial load of vehicle $k$ \\
\cline { 2 - 3 } & $q_{0 j k}$ & The final load of vehicle $k$ \\
\hline
\end{tabular}

The mechanism of environmental measurement is described in the following. The emission of $\mathrm{CO}_{2}$ is caused directly by the consumption of certain type of fuel for vehicles. The $\mathrm{CO}_{2}$ emission rate $(C E R)$ is relative fixed provided that the type of fuel is known, e.g. $2.61 \mathrm{~kg} /$ liter in case of the diesel oil. The fuel consumption rate $(F C R)$ is determined by the travelling distance and the load of vehicles. In order to calculate the $F C R$, we adapt a linear expression between the fuel consumption and the weight of vehicle, and integrate it into our model. The empty-load and full-load fuel consumption rate of vehicles are denoted as $\rho_{0}$ and $\rho^{*}$ respectively. The fuel consumption rate $\rho$ under the load $q$ is expressed as follows.

$$
\rho(q)=\rho_{0}+\frac{\rho^{*}-\rho_{0}}{Q} q
$$

When vehicle $k$ is travelling from customer $i$ to customer $j$, the travelling distance and the load of vehicle $k$ are represented as $d_{i j}$ and $q_{i j k}$ respectively, thus the $\mathrm{CO}_{2}$ emission for vehicle $k$ should be represented as follows.

$$
e_{i j k}=C E R *\left(\rho_{0}+\frac{\rho^{*}-\rho_{0}}{Q} q_{i j k}\right) * d_{i j}
$$

\subsection{Environmental vehicle routing model}

Based on the fundamental vehicle routing model and the new environmental 
consideration described in the above sections, we can provide the complete formulation of the environmental vehicle routing problem (EVRP). Decision variables involved in EVRP are defined as follows.

$x_{i j k}$ is a binary variable, $x_{i j k}=1$ if node $j$ is followed by node $i$ in sequence by vehicle $k$, otherwise $x_{i j k}=0$.

$y_{i k}$ is a binary variable, $y_{i k}=1$ if node $i$ is visited by vehicle $k$, otherwise $y_{i k}=0$.

The complete formulation of EVRP can be represented as follows.

$$
\begin{aligned}
& \min f_{1}=\sum_{i} \sum_{j} \sum_{k} \alpha * d_{i j} * x_{i j k} \\
& \min f_{2}=\sum_{i} \sum_{j} \sum_{k} C E R *\left(\rho_{0}+\frac{\rho^{*}-\rho_{0}}{Q} q_{i j k}\right) * d_{i j} * x_{i j k} \\
& \sum_{i=1}^{n} r_{i} y_{i k} \leq Q, \forall k \\
& \sum_{k=1}^{m} x_{0 j k} \leq m, \forall j \\
& \sum_{k=1}^{m} y_{i k}=1, \forall i \\
& \sum_{i=0}^{n} x_{i j k}=y_{j k}, \forall j,, i \neq j, \forall k \\
& \sum_{j=0}^{n} x_{i j k}=y_{i k}, \forall i, i \neq j, \forall k \\
& q_{0 j k}=\sum_{i=1}^{n} r_{i} y_{i k}, \forall k, j \\
& q_{i 0 k}=0, \forall k, i \\
& q_{i j k}=q_{u i k}-r_{i},(u \rightarrow i \rightarrow j), \forall k \\
& \mathrm{X}=\left(x_{i j k}\right) \in S,(S \subset V \backslash\{0\}) \\
& \sum_{i \in S} \sum_{j \in S} x_{i j k} \leq|S|-1(|S| \geq 2 ; i \neq j ; \forall k) \\
& x_{i j k}=0 \text { or } 1, \forall i, j, k \\
& y_{i k}=0 \text { or } 1, \forall i, j, k
\end{aligned}
$$

Objective (1) is designed to calculate the overall cost aiming to find the optimal routes with minimum economic cost in terms of shortest distance, while objective (2) is to find the optimal routes with minimum environmental cost in terms of the $\mathrm{CO}_{2}$ emission. Constraint (3) indicates that the cumulative customer requirement in one route cannot exceed the maximum capacity of the vehicle. Constraint (4) means the number of vehicles used should be less than the predetermined setting $m$, because some vehicles may be arranged for backup or emergency use. Each customer can only be visited and served by one vehicle under constraint (5). Constraints (6) and (7) 
guarantee the connectivity of each sub-route, which means the visiting vehicle, the departing vehicle and the serving vehicle for one customer can only be the same one. Constraint (8) and (9) describe the initial load and the final load of vehicle $k$ respectively, in which the initial load should equal the cumulative demand of customers associated with this vehicle and the final load should be 0 . While en route, the real-time load of vehicle $k$ can be calculated through the deduction of the demand of its preceding visited customer from its former load, which is expressed in constraint (10) provided that vehicle $k$ visits customers $u, i$ and $j$ sequentially. Constraint (11) and (12) are designated to avoid sub-tours, while the constraint (13) is the binary constraint for the decision variables.

\section{Hybrid Artificial Bee Colony algorithm}

The ABC algorithm belongs to the category of swarm intelligence, which mimics the intelligent behavior of various species, such as ants, birds, bees. Two common distinguishing features of swarm intelligence are self-adjusting and rapid-responsiveness. The $\mathrm{ABC}$ algorithm imitates the behaviors of honeybees, which are divided into three types, scout bees, employed bees and onlooker bees. Different types of bees play different roles in the procedure of exploration and exploitation of food sources. Food sources are regarded as the solutions to specific problems, while the ones with more nectar correspond to better solutions.

The whole procedure of the ABC algorithm can be described as follows. Scout bees are designated to find the initial food sources by carrying out a random search in the search space. Subsequently, employed bees are sent out to exploit the found food sources, and each employed bee matches one food source. During the procedure of exploitation, each employed bee also carries out a neighborhood search and tries to find a better food source nearby. If a better food source is found, the employed bee would abandon the previous food source and exploit the better one. After the completion of the work of all employed bees, they return to the hive and share their information of their associated food sources with onlooker bees waiting in the hive through a waggle dance. The onlooker bees choose to follow certain employed bees and exploit the corresponding food sources probabilistically. This probability is affected mainly by the richness of the corresponding food sources. Once an onlooker bee chooses to follow an employed bee, it becomes an employed bee and repeats the procedure of the employed bees. After certain number of iterations of the procedure of exploitation and exploration, one food source may be exhausted. In that case, the associated employed bee becomes a scout bee and repeating the procedure of scout bees, randomly finding a new food source to replace the abandoned one. The flow chart of $\mathrm{ABC}$ algorithm is described in figure 1 with four periods confirmed in the above description, (1) initialization phase, (2) employed bee phase, (3) onlooker bee 
phase and (4) scout bee phase.

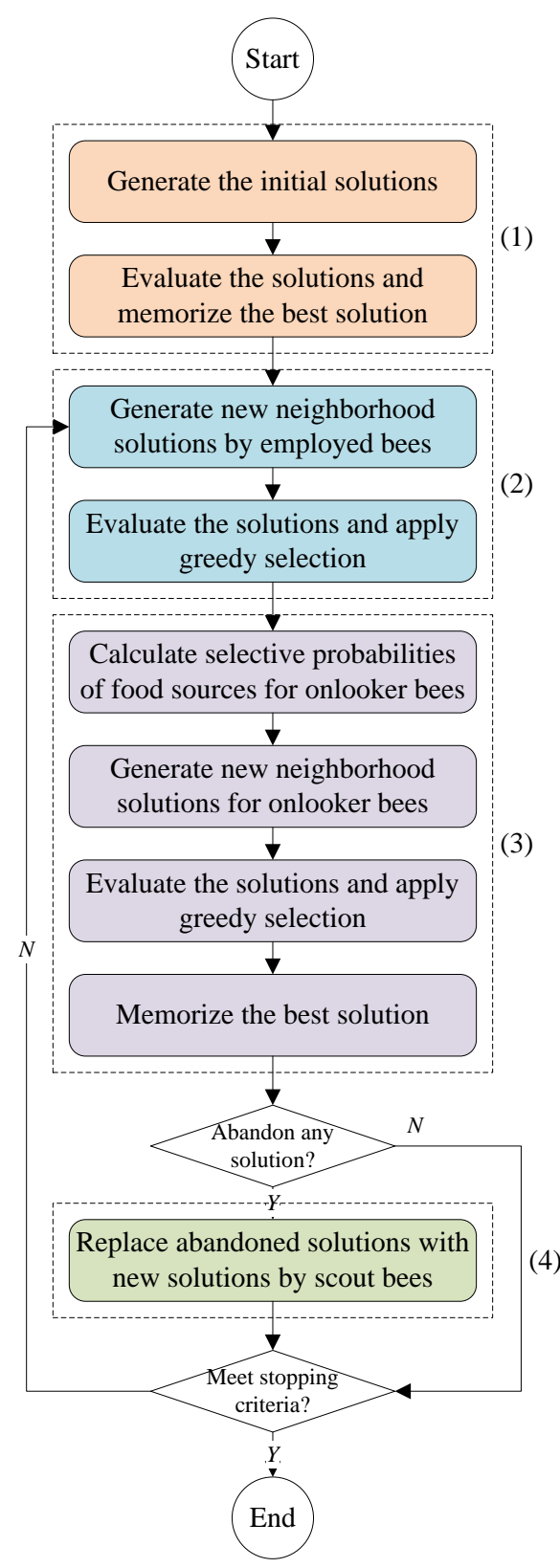

Fig. 1. The flow chart of the $\mathrm{ABC}$ algorithm

Based on the procedures mentioned above, we provide the pseudo code of $\mathrm{ABC}$ algorithm in table 3, which also contains the explanation of the notations used. The ABC algorithm was initially proposed to solve continuous numerical problems without constraints; the generation of initial solutions is determined by the intrinsic range of each dimensional variable referring to equation (1). The neighbor solution is computed through the intersection of the two existing solutions in equation (3); the current solution and another randomly chosen solution. Greedy selection is applied in each neighborhood search which can facilitate the capability of the intensive search, while the abandonment function enhances the capability of the diversified search. 
However, the original $\mathrm{ABC}$ algorithm is not appropriate for the EVRP model due to the different problem contexts, the discrete variable structures and the irregular search space. Apart from that, the required constraints in the proposed model also force the changes of original $\mathrm{ABC}$ algorithm. Therefore, based on the original $\mathrm{ABC}$ algorithm, we propose a hybrid $\mathrm{ABC}$ algorithm for the EVRP model described in the following subsections.

Table 3. The pseudo code of ABC algorithm

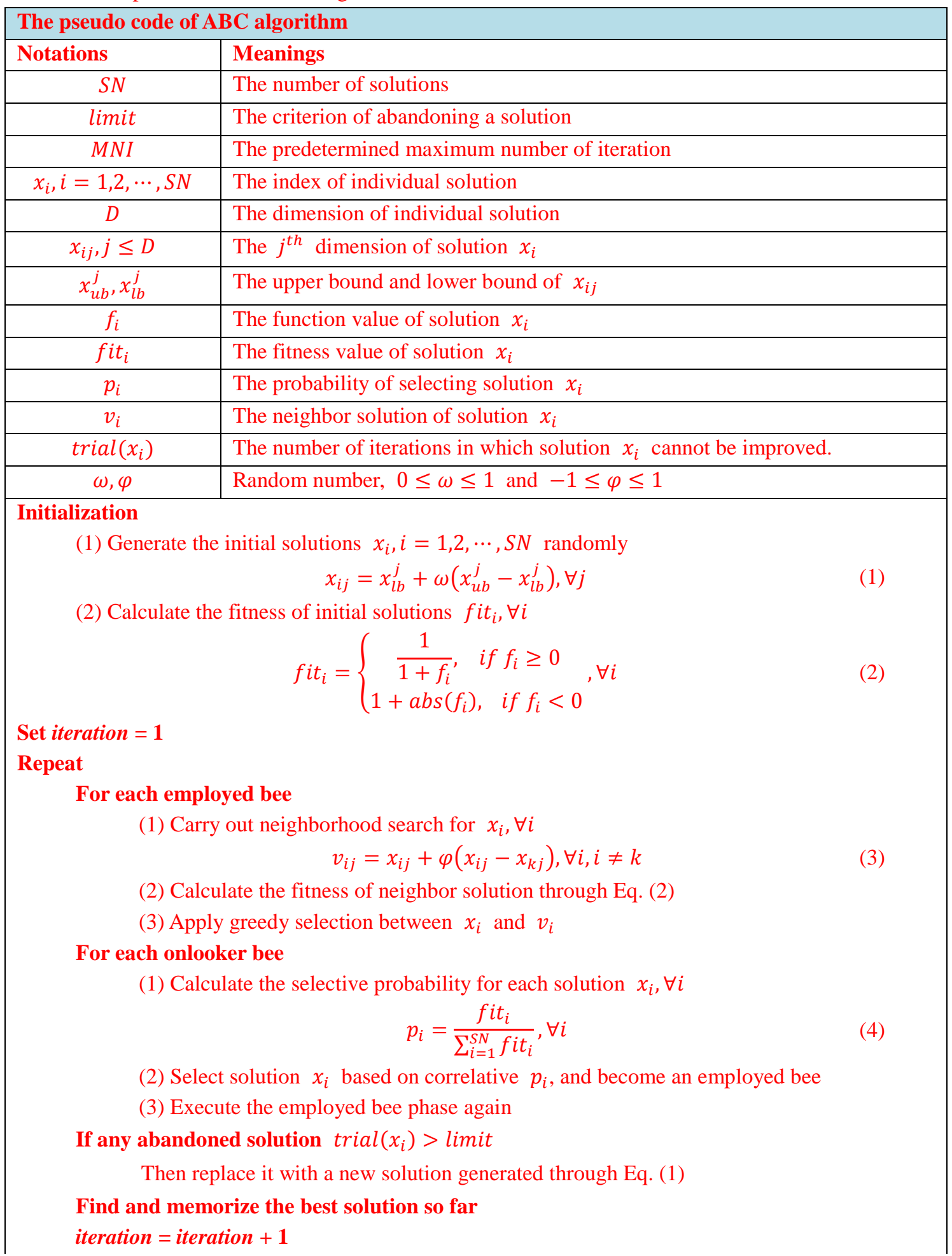




\subsection{Representation scheme and search space}

Since the $\mathrm{ABC}$ algorithm is initially designed for continuous numerical problems, the initial solutions are generated by taking into account the lower and upper bounds of the dimensional variables of a specific problem. However, in CVRP, all dimensions of each solution are related, which means the dimensional variables cannot be generated separately. Therefore, we have to consider all dimensional variables simultaneously in constituting the solution. After an extensive review of the relevant literature, we find two popular representation schemes which are frequently used. The first one is expressed as $X=\left\{X_{v}, X_{r}\right\} . X_{v}$ and $X_{r}$ are the vehicle information and route information associated with each customer respectively. $1 \leq x_{v i} \leq K$ ( $K$ is the number of vehicles, $x_{v i}$ is the vehicle information of customer $i$ ). $1 \leq x_{r i} \leq N$ (N is the number of customers, $x_{r i}$ is the route information of customer $i$ ). The dimension of solution $X$ is $2 N$ (two times of the number of customers). For example, $X_{v}=\{1,2,2,2,2,3,3\}$ and $X_{r}=\{5,4,3,2,7,1,5\}$. Due to the random generation of $X_{r}$, it may have to be re-sorted per vehicle as $X_{r}^{*}=\{1,3,4,1,2,1,2\}$. In this case, the solution contains three sub-routes, $\{0,1,0\},\{0,4,5,2,3,0\}$ and $\{0,6,7,0\}$.

The second one is stated as a random permutation of customers with multiple delimiters, such as 0. Each sub-sequence between two close delimiters is treated as a route. The dimension of this scheme is $N+K+1$. For example, $\mathrm{X}=$ $\{0,1,0,4,5,2,3,0,6,7,0\}$ indicates exactly the same result as the one in first representation scheme. Table 4 illustrates the comparison of the above two representation schemes with a simple example of 7 customers. Compared with the first scheme, the second one is more intuitive and succinct. Not only because of the smaller dimensional scale, the inner generation mechanism of the second scheme can significantly decrease the computational complexity. Thus the second scheme is chosen. The advantages of the first scheme is that it has the potential to handle VRPs with different types of vehicles because the labeled vehicles can be identified

Table 4. Comparison of two representation schemes

\begin{tabular}{|c|c|c|c|c|c|c|c|c|c|c|}
\hline Customer & & & 1 & 2 & 3 & 4 & 5 & 6 & 7 & Solution \\
\hline \multirow{3}{*}{ Scheme 1} & Vehicle & $X_{v}$ & 1 & 2 & 2 & 2 & 2 & 3 & 3 & \multirow{4}{*}{$\begin{array}{l}\text { Route 1: }\{0,1,0\} \\
\text { Route 2: }\{0,4,5,2,3,0\} \\
\text { Route 3: }\{0,6,7,0\}\end{array}$} \\
\hline & Route & $X_{r}$ & 5 & 4 & 3 & 2 & 7 & 1 & 5 & \\
\hline & Updated route & $X_{r}^{*}$ & 1 & 3 & 4 & 1 & 2 & 1 & 2 & \\
\hline Scheme 2 & & \multicolumn{8}{|c|}{$X=\{0,1,0,4,5,2,3,0,6,7,0\}$} & \\
\hline
\end{tabular}

In order to facilitate the process of exploration and exploitation, the search space is not restricted into the feasible region only, which means infeasible solutions are acceptable in the interim states. The infeasible solutions can be used as intermediate solutions to assist the generation of better feasible solutions at next state. In $\mathrm{ABC}$ 
algorithm, given the existing solution, when an employed bee carries out a neighborhood search in the neighborhood area, it may find one solution from the infeasible region. In classical CVRP, when a solution violates the maximum capacity constraint, this solution has to be labeled as an infeasible solution. However, in this research, we use the evaluation mechanism to handle the tolerance of infeasible solutions. For each solution, $x \in \mathrm{X}$, let $p(x)$ denote the total violation of capacity constraints (Alvarado-Iniesta et al., 2013). Referring to the previous notation, $p(x)$ can be expressed as the following.

$$
p(x)=\sum_{k=1}^{m} \max \left\{\sum_{i=1}^{n} r_{i} y_{i k}-Q, 0\right\}
$$

As a result, each solution can be evaluated through the evaluation function as $f(x)=c(x)+\delta * p(x)$. The parameter $\delta$ is self-adjusted, which is gradually enlarged with the increase number of iterations. This allows the existence of infeasible solutions during the searching process but exclude them at the end. The permission and propagation of infeasible solutions during the searching process can substantially raise the exploration and exploitation of the search space.

\subsection{Initialization phase}

In the original $\mathrm{ABC}$ algorithm, the initial solutions are randomly generated by considering the range of dimensional variables. However, this approach can only be used for continuous numerical problems, where the dimensional variables are all real numbers and have a certain range. In this research, a simple and efficient mechanism to generate initial solutions is introduced by using the representation scheme mentioned above. First, we generate a random permutation with a set of customers only, and then split this random permutation of customers into various routes, taking account of the capacity of the vehicles as described in figure 2. Empty routes are also added if necessary to meet the dimensional requirement. Following this mechanism, all the initial solutions can be easily generated and guaranteed to be feasible. It can speed up the convergence of the solutions to a large extent. The guarantee of feasible solutions in the initialization phase does not conflict with the permission and propagation of infeasible solutions in the next states. Another improvement in our designed hybrid $\mathrm{ABC}$ algorithm is that we can run the program multiple times in order to evaluate the average performance. However, the initial solutions are only generated once in the first iteration. Apart from that, the output of last iteration is used as the input of next execution, so the solutions can be continuously improved, which also accelerates the speed of convergence. 


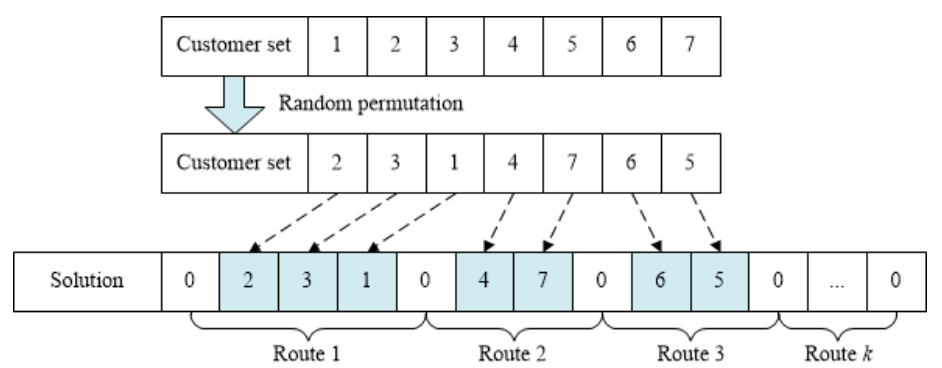

Fig. 2. The generation of initial solutions

\subsection{Employed bee phase}

The initial solutions are generated repetitively following the approach introduced above. Subsequently, employed bees are assigned to exploit the found food sources. One employed bee is assigned to exactly one food source, which means the number of employed bees is the same as the number of food sources. Given the known food source, the corresponding employed bee also tries to search its neighborhood for potential better food sources. In the original $\mathrm{ABC}$ algorithm, the candidate solution is generated through the modification of the current solution by referring to another randomly selected solution. However, when the ABC algorithm is applied into CVRP, this mechanism does not work efficiently. Many researchers have tried to adapt various operators in this procedure to improve the probability of finding better solutions, such as the swap operator, the reverse operator, and the insert operator and

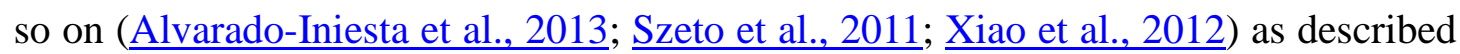
in figure 3.

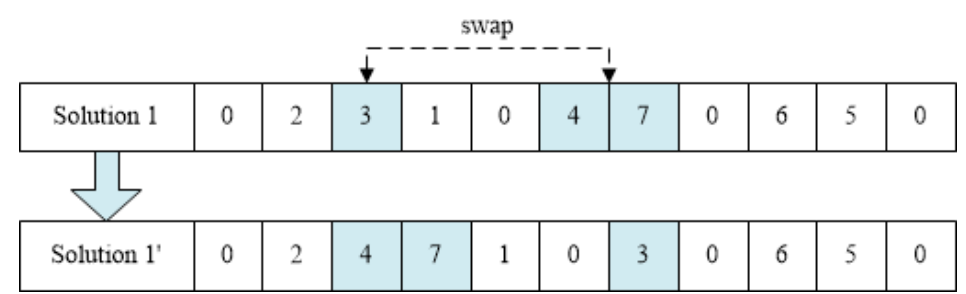

Fig. 3a. Swap operator

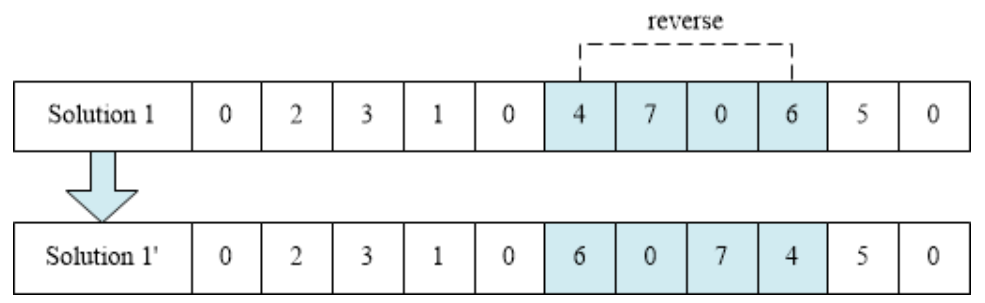

Fig. 3b. Reverse operator 


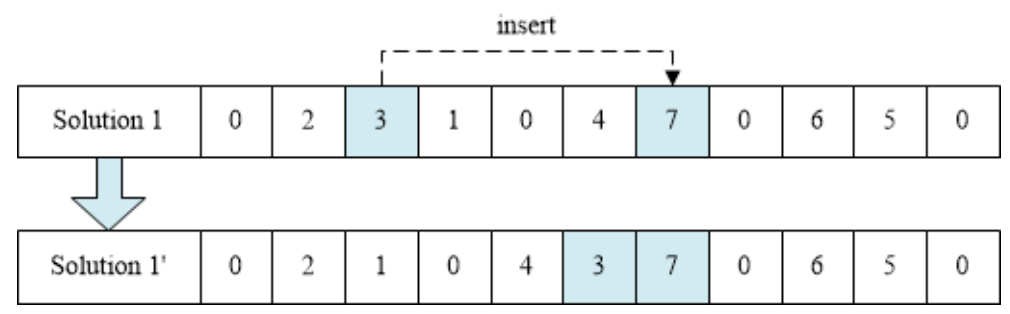

Fig. 3c. Insert operator

The numerical analysis of the efficiency of swap, reverse and insert operators indicates that all of them can assist the evolution of solutions to a certain extent. However, these operators all behave using random searching, which cannot fully utilize the existing information of the current solution. The well-performed sub-routes in the current solution could be easily destroyed by these operators unintentionally. Therefore, in this research, we incorporate the crossover concept from the Genetic Algorithm (GA) (Prins, 2004) into our ABC algorithm . To better utilize the existing information, we choose to use the rule of maximum retention exchange, which is illustrated in figure 4 . The well-performed sub-route from parent solution 1 is selected and reserved in the new solution, while the other parts of this new solution are complemented by parent solution 2, following the rule of maximum retention exchange. Likewise, the well-performed sub-route from parent solution 2 can also be saved and inherited by its child solution. By incorporating this crossover operator, the evolution of solutions can be guided in a good direction and the convergence of solutions can also be increased.

\begin{tabular}{|l|l|l|l|l|l|l|l|l|l|l|l|l|}
\hline Solution 1 & 0 & 2 & 3 & 1 & 0 & 4 & 7 & 0 & 6 & 5 & 0 & 0 \\
\hline
\end{tabular}

Fig. 4. Crossover operator

Another improvement is the adoption of the local search mechanism to each sub-route within the new generated solutions. The approaches of 2-optimization and 3-optimization, which optimize the provided solution through the exchange of two or three elements, are both considered in our research depending on the length of corresponding sub-route. Once a new solution is generated, we apply the local search operator to all valid sub-routes contained in this solution. Essentially, the above swap, reverse, insert and crossover operators are all designed to be applied to the solution level, while the local search operator is adapted in the sub-route level. This means the local search operator can complement the flaws in the randomness of swap, reverse, 
insert and crossover operators and enhance the efficiency of the intensive search around the optimal solution. The efficiency of the local search operator is evaluated and analyzed in our numerical experiments.

\subsection{Onlooker bee phase}

After the completion of the employed bee phase, the employed bees return to the hive and share their food information with onlooker bees. The onlooker bees choose to follow certain employed bees and exploit their corresponding food sources randomly. In the ABC algorithm, the number of onlooker bees is set to be equal to the number of employed bees, and the selective probability is computed through the roulette wheel mechanism. Therefore, the food sources with large amounts of nectar may be selected multiple times, which could promote the propagation of good solutions, and intensify the local search and increase the speed of convergence. For solution $x_{i}$, let $f_{i}$ and $f_{i t} t_{i}$ denote the total cost and fitness of this solution respectively. $f_{i} t_{i}$ can be computed using the Eq. (2) from the original ABC algorithm, while $p_{i}$ is the selective probability of solution $x_{i}$ calculated using Eq. (4). With the known probability, an onlooker bee chooses to exploit a specific food source, and repeats the same procedure as the employed bee phase.

\subsection{Scout bee phase}

Due to iterative exploitation, the nectar in some food sources is gradually consumed and may finally be exhausted. The exhausted food source would be abandoned by its corresponding employed bee. In this case, the associated employed bee becomes a scout bee, and finds a new food source randomly to replace the abandoned one. In the $\mathrm{ABC}$ algorithm, each solution is labeled with one trial number. If one solution cannot be improved after a certain number of trials, it would be abandoned. The procedure is: when a neighborhood search of the current solution is conducted and no better solutions are found, the trial number of the current solution is increased by 1 . However, if a new solution with better fitness is found, it directly replaces the current solution and resets the trial number as 0 . After each onlooker bee phase, the trial numbers of all the solutions will be checked. If the trial number of one solution exceeds a predetermined parameter, this solution would be replaced by a new generated solution in the scout bee phase. As a result of this supplemental scout bee phase, the diversified search ability of the $\mathrm{ABC}$ algorithm is strengthened, which facilitates the convergence of the solutions to a global optimal one. 


\section{Experimental Results}

In the numerical experiment, fifteen benchmark distance-constrained VRP instances from Augerat et al. (1995) and four instances from Christofides and Eilon (1969) are adopted. The names of instances indicate the category of the associated files, the number of nodes on the graph, and the number of vehicles acquired in the optimal solutions. For example, instance "A-n32-k5" indicates that this instance is in category A with 32 nodes, and 5 vehicles are used in the optimal solution. The locations of customers and the depot are provided in a coordinated form. The demand of customers and the capacity of vehicles are predetermined in each instance as well.

The $\mathrm{ABC}$ algorithm provides a rather simple swarm-based optimization technique, in which only two parameters need to be tuned. The first one is the size of the bee colony $(C S)$, which is two times of the number of solutions $(S N)$. The number of employed bees and onlooker bees, which equals the number of food sources, is set to be half of the bee colony size. The second parameter is the criterion of the abandonment of certain solutions (limit). The detailed analysis of parameter $S N$ and limit is provided in the following subsection. As for the parameter $\delta$ employed in the evaluation function as $f(x)=c(x)+\delta * p(x), x \in X$, since the variance of $\delta$ is consistent with the increase of the number of iterations, we calculate $\delta$ by multiplying another positive coefficient $\tau$ and the current index of iterations. In this case, we set $\tau$ to be 0.1 referring to the setting of the maximum number of iterations. The complete parameter settings are provided in table 5. The hybrid ABC algorithm is coded in Java with Eclipse IDE, and all tests are performed on a PC with a $2.5 \mathrm{GHz}$ processor. Each combination of parameters and operators is executed 10 times repetitively, and the average value is used to illustrate the computational performance in order to provide convincing results.

Table 5. Parameter settings for numerical experiment

\begin{tabular}{|c|c|}
\hline Settings & Explanation \\
\hline Instance & $\begin{array}{l}\text { The number of customers is provided in each instance. } \\
\text { The locations of customers and the depot are provided in coordinate format in each } \\
\text { instance. } \\
\text { The customer demands are provided in each instance. } \\
\text { The vehicle capacity is provided in each instance. } \\
\text { The full connectivity among all the nodes on the graph is assumed. } \\
\text { The distance between two nodes, } i\left(x_{i}, y_{i}\right) \text { and } j\left(x_{j}, y_{j}\right) \text {, is calculated as } d_{i j}= \\
\sqrt{\left(x_{i}-x_{j}\right)^{2}+\left(y_{i}-y_{j}\right)^{2}}\end{array}$ \\
\hline Model & $\begin{array}{l}\alpha=1 \text { for the calculation of economic cost in terms of distance } \\
C E R=2.61 \text { The } \mathrm{CO}_{2} \text { emission rate in terms of the diesel oil }\end{array}$ \\
\hline
\end{tabular}




\begin{tabular}{|l|l|}
\hline & $\rho_{0}=0.296$ The empty-load $F C R$ \\
& $\rho^{*}=0.390$ The full-load $F C R$ \\
& $m=2 k$ The maximum number of vehicle available \\
& $\delta=\tau *$ the index of current iteration The penalty coefficient \\
& $\tau=0.1$ \\
\hline Algorithm & $\begin{array}{l}\text { CS The size of be colony } \\
\text { limit } \text { The criterion of abandonment of solutions }\end{array}$ \\
\hline
\end{tabular}

\subsection{Parameter analysis}

As we mentioned, the $\mathrm{ABC}$ algorithm provides a relatively simple mechanism, in which only two parameters need to be tuned, $S N$ and limit. In general, larger size of bee colony can initiate more parallel searches simultaneously and facilitate the diversified exploration. However, the computational time increases substantially because of the size of bee colony, especially when the number of iterations is also large. The limit is normally set to be the product of the number of solutions $(S N)$ and the number of dimensions $(D)$ as limit $=S N * D$. However, in this case, $D$ is replaced with the number of vehicles $(K)$ in one solution as limit $=S N * K$, because the latter one can promote the diversified exploration of the search space better.

The analysis of parameters is conducted with the instance E-n51-k5 first so as to find the best combination, which is displayed in figure 5. The results show that the setting of colony size $(C S)$ as 10 is not sufficient to acquire a good performance. By contrast, the $C S$ setting as 20 is already adequate for an acceptable result; meanwhile it costs less time than the $C S$ setting as 40 and 80 . Once the $C S$ as 20 is determined, we test the performance of the hybrid $\mathrm{ABC}$ algorithm with different settings of the limit. From the figure 5 it is found that the smaller of the limit, the more diversified of the evolution. In this case, we set the limit as $S N^{*} K$ in order to balance the effect of diversification and intensification of the evolution procedure. Thus the combination of $C S$ as 20 and limit as $S N^{*} K$ is eventually employed solving all the instances.

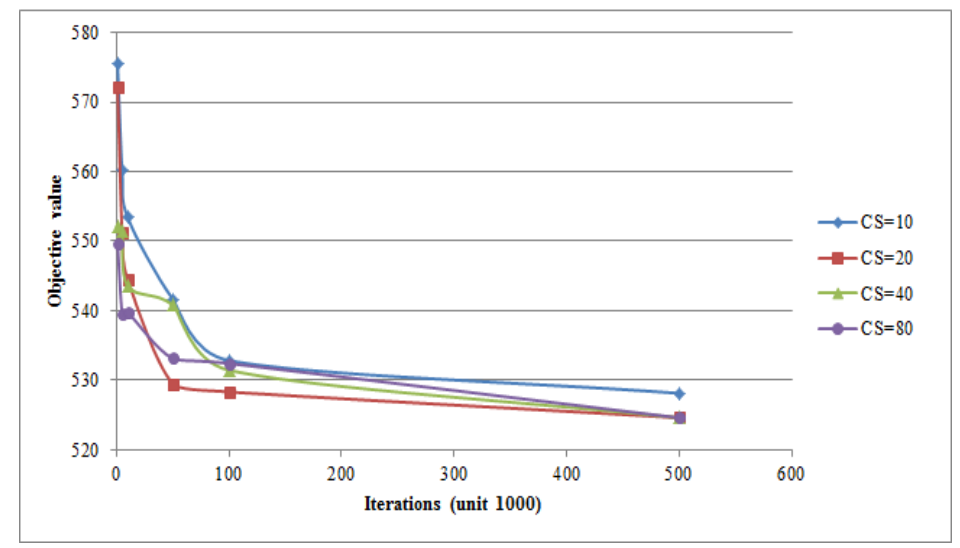

Fig. 5a. The setting of $C S$ 


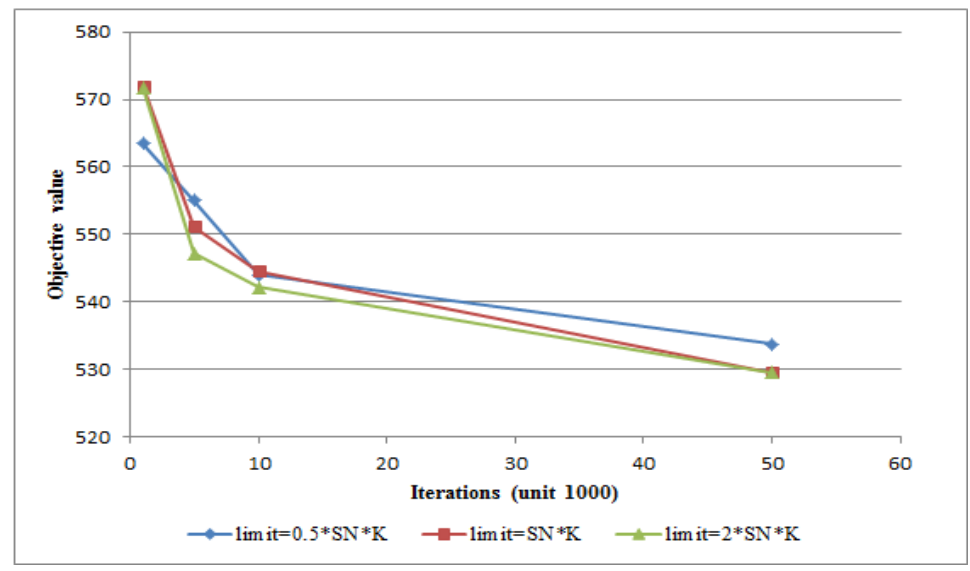

Fig. 5b. The setting of limit

\subsection{Operator analysis}

In the proposed hybrid $\mathrm{ABC}$ algorithm, five operators (i.e. swap operator, reverse operator, insert operator, crossover operator and local search operator) are adopted. Among them, the swap, reverse and insert operators are frequently used in many research studies (Szeto et al., 2011). However, there are no distinguishing strengths and weaknesses among them. Different research provides different efficiency analysis of these three operators, and most of them are based on specific problems. In this case, the above mentioned benchmark instance (i.e. E-n51-k5) is adopted to compare the performance of the three operators. We then incorporate the crossover operator and local search operator into the procedure of neighborhood search, and constitute a hybrid operator by combining all the mentioned operators. The operational performances of swap, reverse, insert and hybrid operators are illustrated in figure 6.

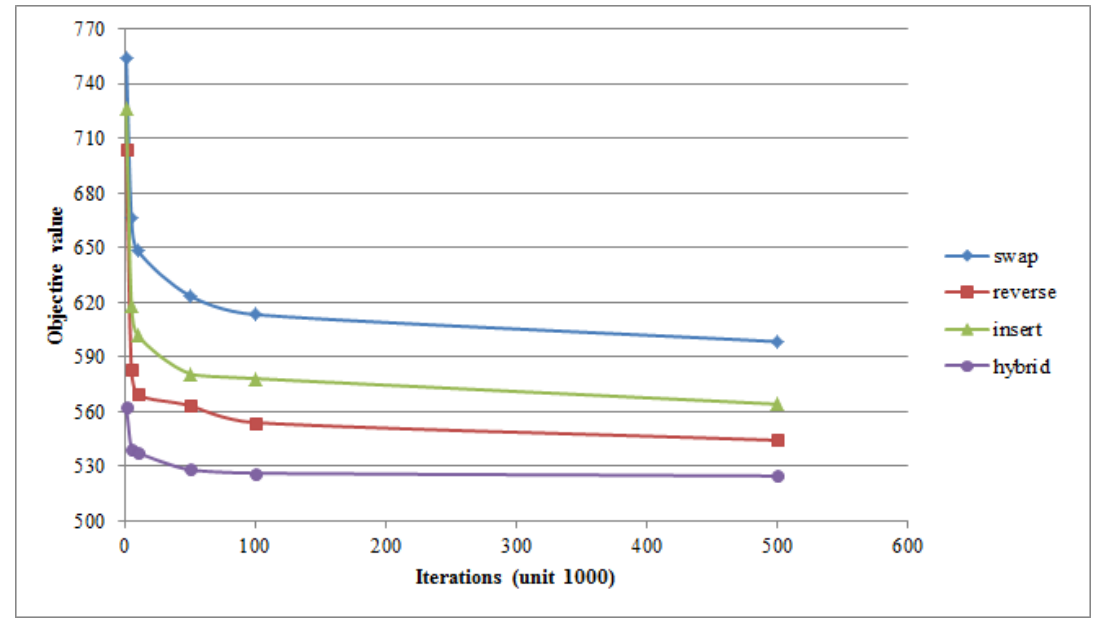

Fig. 6. Comparison of different operators

In the experiment, the reverse operator performs the best with a $9.9 \%$ improvement over the swap operator and a $4.1 \%$ improvement over the insert operator. 
In addition, the insert operator has a $6.0 \%$ improvement over the swap operator. The swap operator performs the worst when we adopt a single operator in the neighborhood search procedure. The results demonstrate that the hybrid operator outperforms the other operators. Especially, when the iteration is small, the hybrid operator even outperforms the reverse operator by $20 \%$. With the increase in the number of iterations, the differentiation of performance is gradually decreased. However, the hybrid operator still performs $3 \%$ better than reverse operator when the number of iterations reaches 500,000. In addition, the results indicate that a single operator can hardly drive the evolution of solutions to a satisfactory convergent point. Comparatively, the hybrid operator works well due to the better utilization of the existing information and the intensive local search by the crossover operator and the local search operator collaboratively.

\subsection{Algorithm performance analysis}

Since the inspiration of the hybrid $\mathrm{ABC}$ algorithm is from the original $\mathrm{ABC}$ algorithm and the GA, we need to evaluate the performance of the proposed hybrid $\mathrm{ABC}$ algorithm through the comparison with the other two. Both the original $\mathrm{ABC}$ algorithm and the GA are adapted to solve the same instance, i.e. E-n51-k5. The parameter settings of GA are as follows. The number of population is 10, which is the same as the number of food sources in $\mathrm{ABC}$ algorithm. The crossover rate and mutation rate are set as 0.9 and 0.1 respectively. $\mathrm{ABC}$ algorithm and $\mathrm{GA}$ are implemented in the same computer using same IDE so as to provide convincing comparison, which is illustrated in figure 7 . From the result we can see that the performance of $\mathrm{GA}$ is like the original $\mathrm{ABC}$ algorithm, and the proposed hybrid $\mathrm{ABC}$ algorithm outperforms them distinctly. The reason why the hybrid $\mathrm{ABC}$ algorithm is over the original $\mathrm{ABC}$ algorithm is certainly due to the incorporation of new operators; the crossover operator and the local search operator. As for the difference between the hybrid $\mathrm{ABC}$ algorithm and the GA could be explained by their own inherent frameworks. For example, the $\mathrm{ABC}$ algorithm comprises four phases, in which the neighborhood search could be conducted twice because of the onlooker bees in one iteration, while in the GA, the generation of new child population could be only once in one loop. 


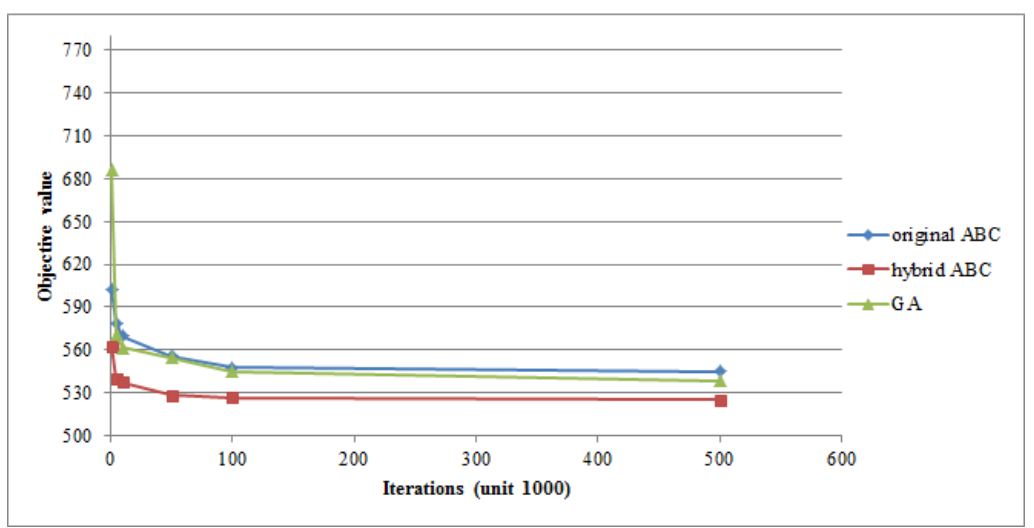

Fig. 7. Comparison of $\mathrm{ABC}$ and GA

\subsection{Optimal solution of EVRP}

In this section, we conduct our experiments for two situations; situation one with the objective of shortest distance and situation two with the objective of the minimum amount of $\mathrm{CO}_{2}$ emission. In order to compare the differentiation of the two optimal solutions, we separately calculate the emission of $\mathrm{CO}_{2}$ along with the optimal solution in situation one and the travelling distance of environmental optimal solution in situation two. Through the analysis of the two situations, it is found that the transformation of the optimal solutions from the shortest travelling distance situation to the minimum $\mathrm{CO}_{2}$ emission situation is the result of the combination of the two factors, travelling distance and load. Even though in the shortest travelling distance situation, the amount of $\mathrm{CO}_{2}$ emission varies due to the different travelling direction starting from the depot.

In the formulation of the environmental objective, three parameters should be predetermined; the $\mathrm{CO}_{2}$ emission rate per liter of fuel, the fuel consumption rate for both empty-load and full-load situations. Numerical experiments indicate that the parameter of the empty-load and full-load affects the weighting of distance and load directly, which further affects the final optimal solution. Referring to a previous case study by Ubeda et al. (2011) CER is set as 2.61, empty-load fuel consumption rate $\rho_{0}=0.296$ and full-load fuel consumption rate $\rho^{*}=0.390$. The results are illustrated in table 6 . Since the instances are all provided in the symmetric matrix format, for a given route, the amount of $\mathrm{CO}_{2}$ emission might be different starting from two opposite directions. $\mathrm{CO}_{2} \_$min and $\mathrm{CO}_{2} \_\max$ are designed to record the minimum and maximum amounts of $\mathrm{CO}_{2}$ emission separately, along with the optimal solution for the situation of shortest travelling distance by considering the influence of two opposite directions starting from the depot. 
From the results, in the situation of the shortest travelling distance, by re-sorting each sub-route within the optimal solution, the amount of $\mathrm{CO}_{2}$ emission could be reduced by $2.47 \%$ on average. The optimal solution in the situation of minimum $\mathrm{CO}_{2}$ emission could further achieve $0.25 \%$ better, on average, which leads to an increase of $0.34 \%$ in total travelling distance comparing with its counterpart in the first situation. In some cases, minimum $\mathrm{CO}_{2}$ emission may have longer distance as $\mathrm{CO}_{2}$ emission does not only depend on traveling distance; it is also affected by the load of the vehicle and traveling direction. The result from table 6 also indicates that the $\mathrm{CO}_{2}$ saving from the changes of travelling direction accounts for a large proportion in the transformation of the optimal solutions from situation one to situation two, which meets our expectation that, in reality, the optimal solutions for two situations should not differentiate too much from each other. In practical situations, the factor of the travelling distance is much more crucial than the load factor, which explains the slight changes from the solution of $\mathrm{CO}_{2} \_$min to the environmental optimal solution.

Table 6. Computational results of numerical experiments

\begin{tabular}{|c|c|c|c|c|c|c|c|c|}
\hline \multirow{2}{*}{ Instance } & \multicolumn{4}{|c|}{ Shortest distance solution } & \multicolumn{2}{|c|}{$\begin{array}{l}\text { Minimum } \mathrm{CO}_{2} \\
\text { solution }\end{array}$} & \multirow{2}{*}{$\begin{array}{l}\mathrm{CO}_{2} \\
\text { saving }\end{array}$} & \multirow{2}{*}{$\begin{array}{l}\text { Distance } \\
\text { increase }\end{array}$} \\
\hline & Distance & $\mathrm{CO}_{2} \_\mathrm{min}$ & $\mathrm{CO}_{2} \_\max$ & $\begin{array}{l}\mathrm{CO}_{2} \\
\text { saving }\end{array}$ & $\mathrm{CO}_{2}$ & Distance & & \\
\hline A-n32-k5 & 787.08 & 685.38 & 707.46 & $-3.12 \%$ & 682.58 & 787.81 & $-0.41 \%$ & $+0.09 \%$ \\
\hline A-n33-k5 & 662.11 & 579.98 & 594.88 & $-2.50 \%$ & 579.20 & 662.40 & $-0.13 \%$ & $+0.04 \%$ \\
\hline A-n34-k5 & 780.94 & 685.79 & 698.46 & $-1.81 \%$ & 683.11 & 790.22 & $-0.39 \%$ & $+1.19 \%$ \\
\hline A-n36-k5 & 802.13 & 704.01 & 719.68 & $-2.18 \%$ & 702.36 & 806.17 & $-0.23 \%$ & $+0.50 \%$ \\
\hline A-n37-k5 & 672.47 & 583.00 & 605.31 & $-3.69 \%$ & 580.69 & 676.26 & $-0.40 \%$ & $+0.56 \%$ \\
\hline A-n38-k5 & 734.18 & 642.89 & 665.35 & $-3.38 \%$ & 642.65 & 735.14 & $-0.04 \%$ & $+0.13 \%$ \\
\hline A-n39-k5 & 828.99 & 730.20 & 743.16 & $-1.74 \%$ & 727.33 & 837.07 & $-0.39 \%$ & $+0.98 \%$ \\
\hline A-n46-k7 & 917.91 & 810.73 & 825.54 & $-1.79 \%$ & 803.73 & 926.50 & $-0.86 \%$ & $+0.94 \%$ \\
\hline A-n48-k7 & 1074.34 & 944.29 & 968.14 & $-2.46 \%$ & 944.01 & 1075.16 & $-0.03 \%$ & $+0.08 \%$ \\
\hline B-n31-k5 & 676.09 & 585.19 & 588.83 & $-0.62 \%$ & 585.19 & 676.09 & $-0.00 \%$ & $+0.00 \%$ \\
\hline B-n35-k5 & 956.29 & 839.60 & 851.16 & $-1.36 \%$ & 838.91 & 962.24 & $-0.08 \%$ & $+0.62 \%$ \\
\hline B-n38-k6 & 808.70 & 698.50 & 726.05 & $-3.79 \%$ & 698.50 & 808.70 & $-0.00 \%$ & $+0.00 \%$ \\
\hline B-n39-k5 & 553.16 & 484.67 & 495.37 & $-2.16 \%$ & 480.88 & 553.27 & $-0.78 \%$ & $+0.02 \%$ \\
\hline B-n43-k6 & 746.98 & 652.50 & 676.07 & $-3.49 \%$ & 651.52 & 750.41 & $-0.15 \%$ & $+0.46 \%$ \\
\hline B-n44-k7 & 914.96 & 805.66 & 825.29 & $-2.38 \%$ & 804.94 & 915.18 & $-0.09 \%$ & $+0.02 \%$ \\
\hline E-n22-k4 & 375.28 & 325.63 & 340.34 & $-4.32 \%$ & 325.63 & 375.28 & $-0.00 \%$ & $+0.00 \%$ \\
\hline E-n23-k3 & 568.56 & 481.50 & 494.29 & $-2.59 \%$ & 479.02 & 569.75 & $-0.52 \%$ & $+0.21 \%$ \\
\hline E-n33-k4 & 837.67 & 735.69 & 750.54 & $-1.98 \%$ & 734.88 & 837.87 & $-0.11 \%$ & $+0.02 \%$ \\
\hline E-n51-k5 & 524.61 & 464.17 & 471.26 & $-1.51 \%$ & 463.19 & 527.50 & $-0.21 \%$ & $+0.55 \%$ \\
\hline Average & & & & $-2.47 \%$ & & & $-0.25 \%$ & $+0.34 \%$ \\
\hline
\end{tabular}

The instance of E-n51-k5 exemplifies the specific differentiation of two optimal solutions in corresponding situations. The best known solution for this instance, with 


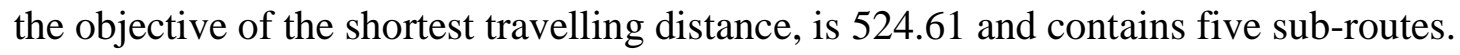
Each sub-route is bidirectional since the source data is provided in a symmetric matrix. Thus we need to calculate the maximum and minimum amount of $\mathrm{CO}_{2}$ emission along with the optimal solution of the shortest travelling distance. After that, we apply our new EVRP model to this instance. All the results are described in table 7. From the results, it is found that the number of routes is the same for both situations. The first, second and forth sub-routes are exactly the same. For the third and fifth sub-routes, the contained nodes are also the same. However, the sequences are quite different, which means even though the overall travelling distance is increased by $0.51 \%$, the construction of the sub-routes could be largely changed. Figure 8 shows the differentiation of the two optimal solutions.

Table 7. Optimal results for instance with 50 customer nodes

\begin{tabular}{|c|c|c|c|}
\hline & Distance & $\mathrm{CO}_{2}$ & Optimal solution \\
\hline \multirow{2}{*}{$\begin{array}{l}\text { Situation } 1 \\
\text { Shortest distance }\end{array}$} & \multirow{2}{*}{524.61} & $\begin{array}{l}\text { (a) } \\
\mathrm{CO}_{2} \_\max \\
471.26\end{array}$ & 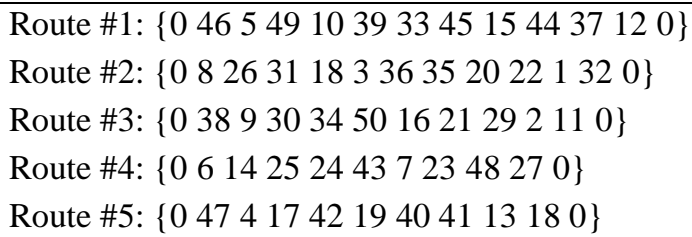 \\
\hline & & $\begin{array}{l}\text { (b) } \\
\mathrm{CO}_{2 \_} \text {min } \\
464.17\end{array}$ & 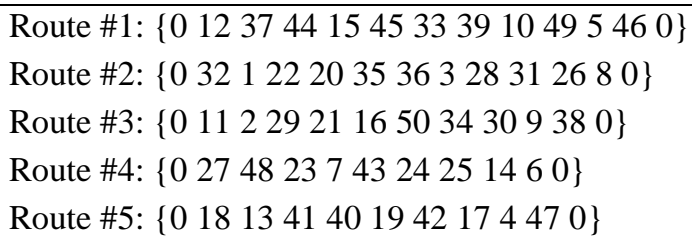 \\
\hline $\begin{array}{l}\text { Situation } 2 \\
\text { Minimum emission }\end{array}$ & 527.50 & $\begin{array}{l}(\mathrm{c}) \\
\mathrm{CO}_{2} \\
463.17\end{array}$ & 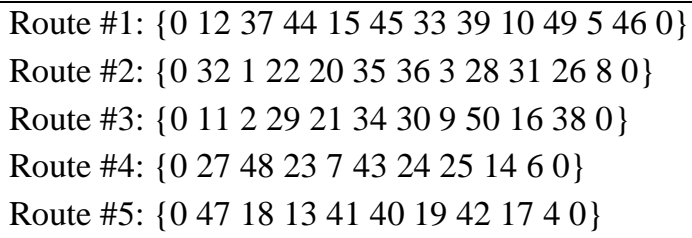 \\
\hline
\end{tabular}

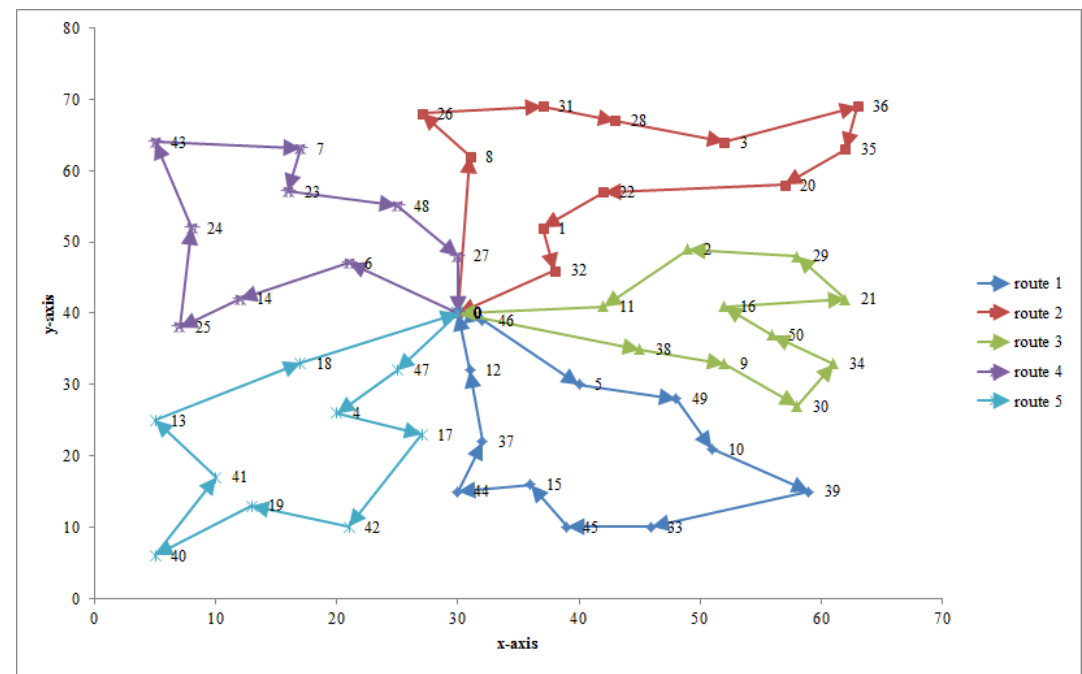

Fig. 8a. Optimal solution of the shortest travelling distance with $\mathrm{CO}_{2}$ max 


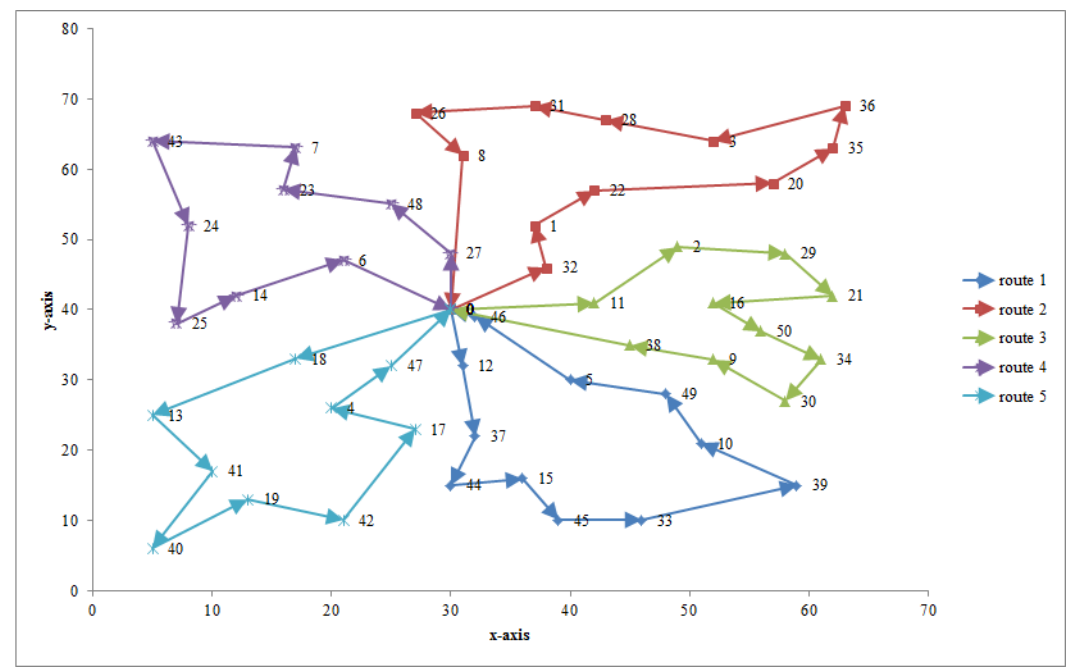

Fig. 8b. Optimal solution of the shortest travelling distance with $\mathrm{CO}_{2}$ min

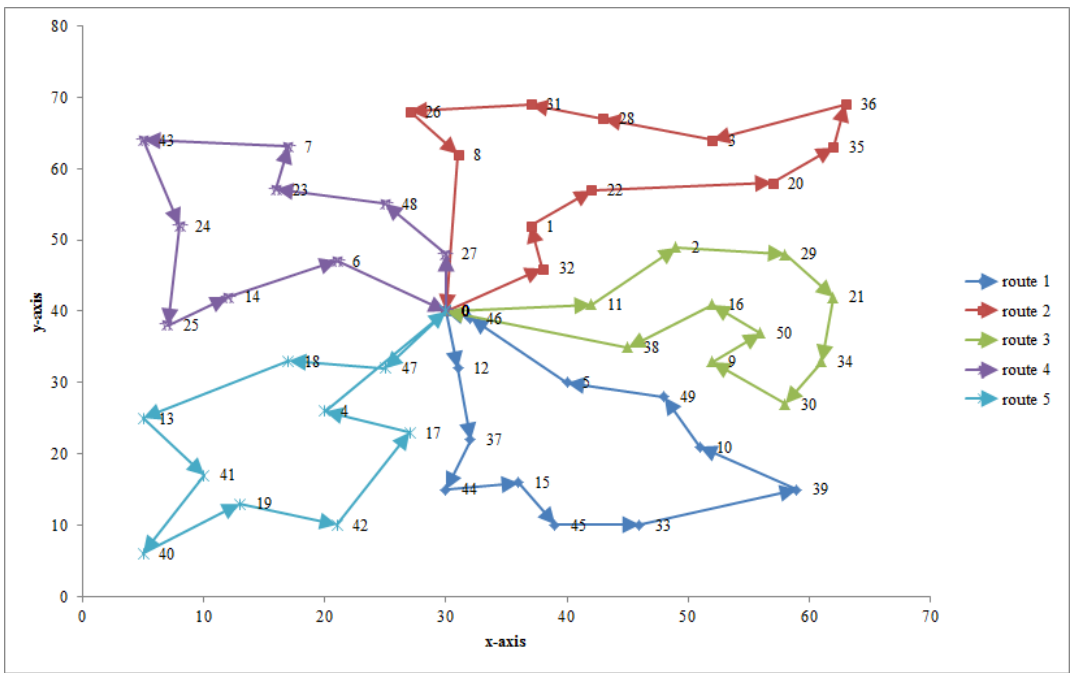

Fig. 8c. Optimal solution of the minimum $\mathrm{CO}_{2}$ emission

\section{Conclusions and future works}

This research examines the vehicle routing problem from an environmental perspective and proposes a new model of environmental vehicle routing problem (EVRP), which meets the latest requirements in practical. The emission of carbon dioxide $\left(\mathrm{CO}_{2}\right)$ is used to measure the environmental impact, alongside way the vehicle routing. The emission of $\mathrm{CO}_{2}$ is caused by the consumption of different types of fuel or energy, and may also be affected by various factors. In our research, through a comprehensive analysis of various factors applied in practical situations, we find two factors, the load and the travelling distance of vehicles, are the most common and practical factors as opposed to other factors, such as the vehicle speed, road condition, weather and traffic. The other factors are nearly impractical and unreasonable to be measured because the traffic situations in different cities or regions vary significantly 
from time to time. A new EVRP model was constructed with the objective of minimizing the environmental influence by taking these two factors into account. The computational results in our numerical experiments indicate that the optimal environmental solution is meaningful and could likely be accepted by logistics enterprises, even though the overall travelling distance accompanying this solution is slightly increased, in contrast with the known shortest distance. In this research, a hybrid $\mathrm{ABC}$ algorithm was adopted to solve this problem, and was shown to be improved in four aspects; new mechanism of initialization, incorporation of new operators, more suitable adjustment of parameters and continuous improvement. These improvements could either speed up the convergence or promote the diversification of the evolution of solutions. Numerical experiments proved that this hybrid $\mathrm{ABC}$ algorithm outperforms the original $\mathrm{ABC}$ significantly.

Further research should be conducted in at least two directions. Foremost, we could extend the concept of environmental measurement into other VRP variants, such as VRPTW, VRP with multiple types of vehicles, VRP with simultaneously pick-up and delivery and multi-depot VRP. Different variants of VRP may have different considerations in quantifying the environmental influence, and the corresponding factors, such as time window and multi-depots, may have varied influence on the construction of optimal solutions. Secondly, we could apply this new model into practical cases, especially with the actual geographical distribution of customers and depots, and accurate information on the fuel consumption of particular vehicles in order to test the performance for further improvement.

\section{Acknowledgements}

This work is supported by the Hong Kong Polytechnic University. Our gratitude is also extended to the research committee and the Department of Industrial and Systems Engineering of the Hong Kong Polytechnic University for support in this project (\#4-RTY0) and (H-ZD88)

\section{References}

Akay, B., Karaboga, D., 2012. Artificial bee colony algorithm for large-scale problems and engineering design optimization. J Intell Manuf 23(4), 1001-1014.

Akyelken, N., 2011. Green Logistics: Improving the Environmental Sustainability of 
Logistics. Transport Reviews 31(4), 547-548.

Alvarado-Iniesta, A., Garcia-Alcaraz, J.L., Rodriguez-Borbon, M.I., Maldonado, A., 2013. Optimization of the material flow in a manufacturing plant by use of artificial bee colony algorithm. Expert Systems with Applications 40(12), 4785-4790.

Augerat, P., Belenguer, J., Benavent, E., Corberán, A., Naddef, D., Rinaldi, G., 1995. Computational results with a branch and cut code for the capacitated vehicle routing problem. Rapport de recherche- IMAG.

Blum, C., Li, X., 2008. Swarm Intelligence in Optimization, In: Blum, C., Merkle, D. (Eds.), Swarm Intell. Springer Berlin Heidelberg, pp. 43-85.

Blum, C., Merkle, D., 2008. Swarm intelligence: introduction and applications. Springer.

Bochtis, D.D., Sørensen, C.G., 2010. The vehicle routing problem in field logistics: Part II. Biosystems Engineering 105(2), 180-188.

Bonabeau, E., Dorigo, M., Theraulaz, G., 1999. Swarm intelligence: from natural to artificial systems. Oxford university press New York.

Christofides, N., Eilon, S., 1969. An Algorithm for the Vehicle-Dispatching Problem. OR 20(3), 309-318.

Dobers, K., Röhrig, R., Rüdiger, D., Schneider, M., 2013. Green Logistics: Comparability of the Environmental Effects of Logistics Services, In: Clausen, U., Hompel, M., Klumpp, M. (Eds.), Efficiency and Logistics. Springer Berlin Heidelberg, pp. $135-148$.

Elhedhli, S., Merrick, R., 2012. Green supply chain network design to reduce carbon emissions. Transportation Research Part D: Transport and Environment 17(5), 370-379.

Golden, B.L., Raghavan, S., Wasil, E.A., 2008. The vehicle routing problem: latest advances and new challenges. Springer.

Hoff, A., Andersson, H., Christiansen, M., Hasle, G., Løkketangen, A., 2010. Industrial aspects and literature survey: Fleet composition and routing. Computers \& Operations Research 37(12), 2041-2061.

Jeanne, R., 1986. The evolution of the organization of work in social insects. Monitore zoologico italiano 20(2), 119-133.

Kara, İ., Kara, B., Yetis, M.K., 2007. Energy Minimizing Vehicle Routing Problem, In: Dress, A., Xu, Y., Zhu, B. (Eds.), Combinatorial Optimization and Applications. Springer Berlin Heidelberg, pp. 62-71.

Karaboga, D., 2005. An idea based on honey bee swarm for numerical optimization. Karaboga, D., Ozturk, C., Karaboga, N., Gorkemli, B., 2012. Artificial bee colony programming for symbolic regression. Information Sciences 209(0), 1-15.

Laporte, G., 1992. The vehicle routing problem: An overview of exact and approximate algorithms. European Journal of Operational Research 59(3), 345-358.

Lin, C., Choy, K.L., Ho, G.T.S., Chung, S.H., Lam, H.Y., 2013. Survey of Green Vehicle Routing Problem: Past and future trends. Expert Systems with Applications(0). Prins, C., 2004. A simple and effective evolutionary algorithm for the vehicle routing problem. Computers \& Operations Research 31(12), 1985-2002.

Salimifard, K., Shahbandarzadeh, H., Raeesi, R., 2012. Green Transportation and the 
Role of Operation Research, 2012 International Conference on Traffic and Transportation Engineering.

Sheu, J.-B., Chou, Y.-H., Hu, C.-C., 2005. An integrated logistics operational model for green-supply chain management. Transportation Research Part E: Logistics and Transportation Review 41(4), 287-313.

Suzuki, Y., 2011. A new truck-routing approach for reducing fuel consumption and pollutants emission. Transportation Research Part D: Transport and Environment 16(1), 73-77.

Szeto, W.Y., Wu, Y., Ho, S.C., 2011. An artificial bee colony algorithm for the capacitated vehicle routing problem. European Journal of Operational Research 215(1), 126-135.

Tancrez, J.-S., Lange, J.-C., Semal, P., 2012. A location-inventory model for large three-level supply chains. Transportation Research Part E: Logistics and Transportation Review 48(2), 485-502.

Toth, P., Vigo, D., 2002. An overview of vehicle routing problems. The vehicle routing problem 9, 1-26.

Ubeda, S., Arcelus, F.J., Faulin, J., 2011. Green logistics at Eroski: A case study. International Journal of Production Economics 131(1), 44-51.

Wu, H.-J., Dunn, S.C., 1995. Environmentally responsible logistics systems. International Journal of Physical Distribution \& Logistics Management 25(2), 20-38.

Xiao, Y., Zhao, Q., Kaku, I., Xu, Y., 2012. Development of a fuel consumption optimization model for the capacitated vehicle routing problem. Computers \& Operations Research 39(7), 1419-1431. 


\section{University Library}

\section{- M M I N E R VA A gateway to Melbourne's research publications}

Minerva Access is the Institutional Repository of The University of Melbourne

Author/s:

Zhang,;Lee,;Choy,;Ho, W;Ip,

Title:

Design and development of a hybrid artificial bee colony algorithm for the environmental vehicle routing problem

Date:

2014-08

Citation:

Zhang, , Lee, , Choy, , Ho, W. \& Ip, (2014). Design and development of a hybrid artificial bee colony algorithm for the environmental vehicle routing problem. Transportation Research Part D: Transport and Environment, 31, pp.85-99. https://doi.org/10.1016/j.trd.2014.05.015.

Persistent Link:

http://hdl.handle.net/11343/118654 